\title{
Estudio metabólico en el lactante desnutrido alimentado con una nueva dieta de bajo contenido en leche
}

\author{
ORES.: JORGE MARTNER G.*, SERGIO JARPA Y. ${ }^{*}$, FERNANDO MONCKEBERG $B_{\mathrm{t}}$ ***
}

\begin{abstract}
Aún hoy día, el tratamiento del lactante con desnutrición grave no ha sido enteramente resuelto, y es asi como aun en los mejores servicios hospitalarios la mortalidad es muy elevada y el tiempo requeriđo para la recuperación es muy largo. Por una parte son frecuentes los trastornos digestivos intercurrentes, que complican y retardan la recuperación y por otra, aun en ausencia de complicaciones infecciosas $\mathrm{y}$ recibiendo una alimentación teóricamente adecuada, estos enfermos demoran largos períodos de tiempo antes de reiniciar la recuperación.
\end{abstract}

Han sido descritos numerosos trastornos metabólicos, que explican la falta de respuesta al tratamiento, y que son la consecuencia del proceso adaptativo a la subalimentación crónica. Han sido descritas también diversas alteraciones en los procesos de absorción intestinal, que impiden un aprovechamiento de los diversos nutrientes y que pueden ser la causa de los trastornos digestivos que se observan frecuentemente durante el tratamiento. Es asi como se ha descrito alteraciones en la absorción de ciertos hidratos de carbono y también de grasas.

Tradicionalmente se ha prescrito para el tratamiento del lactante desnutrido, al igual que el niño normal, fórmulas en base de leche humana o de vaca. Sin embargo, en el caso del nin̄o desnutrido no parece ser lo más adecuada tanto por la composición de los hidratos de carbono como de los ácidos grasos de la leche.

El único hidrato de carbono de la leche está constituido por un disacárido (lactosa), que para

\footnotetext{
* Laboratorio de Investigación y Docente de Pediatría Area Oriente.

* Servicjo de Nutrición, Unidad Hospital "Luis Calvo Mac. kenna".

* a* Departamento de Nutrición y Techologia de Aljmentos. Sede

Sur, Universidad de Chile.

* Financiado en parte par Grant NIH HDO 3741-12 y Funda-
}

su absorción y metabolización necesita ser hidrolizado mediante una enzima intracelular específica, la lactosa. En el lactante desnutrido ha sido descrita una intolerancia a la lactosa, que podría explicar la alta frecuencia de diarrea que se observa durante el tratamiento con fórmulas en base de leche en estos enfermos; al dar una sobrecarga de lactosa, no se observa una elevación de la glicemia como sucede en lactantes normales (1) y la determinación directamente en la mucosa yeyunal da valores muy bajos, lo que no sucede con otros disacáridos, como maltosa y sucrosa (1). Estos antecedentes justifican el ensayo de mezclas almenticias en que la lactosa se reemplace total o parcialmente por otros disacáridos.

Por otra parte, la absorción de la grasa de la leche también se encuentra disminuida (3), to que puede explicarse tanto por una disminución de la síntesis de proteínas indispensables para la formación de quilomicrones a nivel de la célula de la mucosa yeyunal.

Como la absorción de grasas depende de la longitud de la cadena de ácidos grasos, como del nivel de saturación (4), parece también aconsejable reemplazar la grasa de la leche por grasas vegetales, que aparecen absorberse mejor (5).

Teóricamente, puede reemplazarse la lactosa por otro hidrato de carbono cuya hidrólisis de otro disacárido que no sea la lactosa. La mayor parte de los hidratos de carbono de la dieta humana se encuentran en la naturaleza en forma de almidones. Estos son perfectamente digeribles por el niño mayor y el adulto, sin embargo, son digeridos con dificultad por el lactante pequeño (6). Por esta razón es aconsejable proporcionar los almidones en forma predigerida, lo que se consigue a través del proceso de extensión, en que mecánicamente se produce la hidrólisis del almidón has- 
ta dextrinas y disacáridos (7) obteniéndose al mismo tiempo una mejor suspensión en agua del producto.

En el presente trabajo se estudió el metabolismo de Nitrógeno, agua, minerales, grasas y crecimiento en lactantes desnutridos de III. Grado.

Las características principales de la fórmula alimenticia utilizada en el tratamiento de estos lactantes cumple con los siguientes objetivos:

a) Disminuir la cantidad de lactosa.

b) Sustituir las grasas por grasas vegetales (maíz).

c) Proporcionar un aporte proteico cuya composición aminoácida asegure un alto valor biológico.

d) Que no presente dificultades en su proporción culinaria y que tenga una dilución y suspensión adecuada.

e) Bajo costo de producción.

Méródo. Se utilizaron 8 lactantes menores de 1 año de edad (entre 4 y 11 meses). Todos ellos desnutridos en III Grado (peso actual inferior al $60 \%$ del peso ideal) con pesos que fluctuaron entre 2.740 y $5.180 \mathrm{Kg}$.

A los lactantes, una vez tratado el cuadro agudo que motiyó su hospitalización, se les sometió a la dieta de recuperación de uso corriente en el hospital, a base de leche descremada acidificada, con adición de una fritura de harina y mantequilla (8), por un período mínimo de 15 días, hasta que iniciaron una ganancia de peso mantenida y constante sin presentar cuadros de infecciones intercurrentes. Al final de este periodo se colocaron en balance metabólico de 5 días con la técnica descrita (9). Al finalizar este período se cambió su alimentación de a una mamadera diaria por la alimentación experjmental. Finalizado el cambio se esperó un tiempo mínimo de 15 días antes de efectuar un $\Pi$ balance con esta nueva dieta. (el tiempo transcurrido entre los dos balances fue entre 15 y 39 días). Sólo uno de ellos presentó un cuadro intercurrente broncopulmonar, lo que obligó a esperar un período de 60 días entre ambos balances). Todos ellos subieron de peso regularmente y no presentaron cuadros intercurrentes antes del período de balance.

Durante toda la hospitalización se les pesó y controló su temperatura diariamente.

El manejo y técnica de análisis de las muestras han sido ya descritas en publicaciones anteriores (9) con la diferencia que el $\mathrm{Na}$ y $\mathrm{K}$. son leídos en un fotómetro de llama Coleman, que funciona con una mezcla de gas butano y propano y $\mathrm{O}_{2}, \mathrm{y}$ el $\mathrm{pH}$ y equilibrio ácido base que son determinados en un aparato Astrup A Ml.
A ellas sólo habría que agregar la medición del $\mathbf{N}$. Uréico, que se practicó con la técnica de la ureasa descrita por Donough O'Brien y Frank Ibbot (10). La creatina en la orina se midió con una técnica modificada de Hare (11) (12) (13) en 3 colecciones separadas de orina, 2 de 24 horas y una de 72 horas en cada balance ( 5 días en total). Los resultados de estos análisis demuestran constancia en la eliminación diaria de este elemento independientemente de la diuresis.

Al utilizar la cifra de creatinina excretada en 24 horas como índice de masa muscular (14) se pudo comprobar variación de ella en períodos de tiempo relativanente cortos.

Las alimentaciones utilizada para esta experiencia fueron las siguientes:

\section{PERIODO CONTROL:}

Fel, Pr: Cant. (g) Prot. Grasas H.de C.

\begin{tabular}{lrrrrr}
\hline Eledón & 7 & 2,0 & 0,9 & 3,2 & \\
Harina & 3 & 0,2 & 0,0 & 2,3 & \\
Aceite & 3 & - & 3,0 & - & \\
Azúcar & 5 & - & - & 5,0 & \\
H20 hasta & & & & & Cal. \\
completar & 100 & & & & \\
\hline Tot a 1 & 100 & 2,2 & 3,9 & 10,5 & $\mathbf{8 6}$
\end{tabular}

Esta alimentación administra 86 calorías por 100 g., $10 \%$ de ellas aportadas por proteínas, $41 \%$ por grasas y $49 \%$ por Hidratos de Carbono. Se prescribió como promedio entre $180-200 \mathrm{~g} /$ $\mathrm{Kg} /$ día.

\section{Periodo Experimental;}

Alimentación Fortesán, alimento preparado por el Laboratorio de Investigaciones Pediátricas U. de Chile cuya composición es:

Cant. Prot. Grasas H.de C. Cal.

\begin{tabular}{llrrrr}
\hline Harina Trigo & 40 & 3,2 & 0,5 & 30,7 & 136,4 \\
Harina Soya & 35 & 12,5 & 1,2 & 14,8 & 114,1 \\
Leche descremada & 25 & 8,8 & 0,3 & 13,0 & 92,0 \\
\hline T o t a l & 100 g. & 24,5 & 2,0 & 58,5 & 342,5
\end{tabular}

Este polvo se utilizó en una dilución de $12 \%$, adicionada de Maltosa Dextrina y aceite de la siguiente manera: 
T A B L A 1

Cant. (g) Prot. Grasas H. de C. Cal.

\begin{tabular}{lrrrrr}
\hline Fortesán & 12,0 & 2,9 & 0,2 & 7,0 & \\
$\begin{array}{l}\text { Aceite (Mezcla) } \\
\text { Maltosa dextrina }\end{array}$ & 6,0 & - & 3,5 & - & \\
$\begin{array}{l}\text { H20 hasta } \\
\text { completar }\end{array}$ & 100 & & & & \\
\hline To t a l & 100 & 2,9 & 3,7 & 13,0 & 96,9
\end{tabular}

Esta alimentación administra 96,9 calorías por 100 g., $12 \%$ aportadas por proteínas, $34 \%$ por grasas y $54 \%$ por Hidratos de Carbono. Se prescribió en promedio entre 130 y $150 \mathrm{~g} / \mathrm{Kg} /$ día.

Resultados. 1. La aceptabilidad de Jas dos alimentaciones fue excelente. No hubo rechazo de parte de ninguna de ellas.

2. La ganancia de peso fue regular y estable, en períodos de tiempo de 20 días para cuatro de ellos, 17 en tres y uno de 15 días. Los promedios de alza fueron de $6,7 \mathrm{~g} / \mathrm{Kg} /$ día para el período control y de $6,7 \mathrm{~g} / \mathrm{Kg} / \mathrm{día}$ para la alimentación experimental. Esta diferencia no fue significativa, (p. $0,24)$.

3. El volumen de ingestión promedio de alimento para los períodos descritos fue de: $173 \mathrm{~g}$ / $\mathrm{Kg} /$ día (142-187) para la dieta control y de: 141 $\mathrm{g} / \mathrm{Kg} /$ día (129-159) para el período experimental.

Balance metabólico. Durante el período de balance ( 5 días) todos subieron de peso en forma estable. El promedio del alza fue de $8,8 \mathrm{~g} /$ $\mathrm{Kg} /$ día para el balance control y $6,6 \mathrm{~g} / \mathrm{Kg} /$ día para el balance experimental. Esta diferencia no fue significativa (p. 0,19 ).

La jngestión promedio de las fórmulas para estos períodos fue de $172,7(137-184) \mathrm{g} / \mathrm{Kg} / \mathrm{día}$ para el control y de $139,8(130-154) \mathrm{g} / \mathrm{Kg} /$ día para la dieta experimental.

El volumen de agua ingerido como tal ascendió a $43 \mathrm{ml} / \mathrm{Kg} /$ día con alimentación control y de $28 \mathrm{ml}$. con Fortesán.

Si se expresa al Nitrógeno retenido como porcentaje del Nitrógeno absorbido se obtiene un $20 \%$ para el de control y un $37 \%$ para el experimental. La diferencia de Nitrógeno retenido es significatica $(\mathrm{p}<0,009)$ (Tabla 1).

El volumen de agua de la orina representa un $47 \%$ del líquido ingerido en el período control y ил $26 \%$ en el experimental (Tabla 3 ).

Este diferente volumen urinario se tradujo en Ias siguientes modificaciones de concentración promedio (Tabla 4).

BALANCE NITROGENADO EN 8 NIÑOS CON DESNUTRICION MARASMICA GRAVE (Mg/Kg/día)

\begin{tabular}{lll}
\hline & Control & Experimental \\
\hline N. Ingerido & 528 & 610 \\
N. Deposiciones & 110 & 133 \\
N. Absorbido & $418(79 \%)$ & $477(78 \%)$ \\
N. Orina & 333 & 300 \\
N. Retenido & $85(16 \%)$ & $177(29 \%)$ \\
\hline
\end{tabular}

T A B L A 2

BALANCE GRASO EN \& NINOS CON DESNUTRICION MARASMICA GRAVE (g/Kg/dia)

Control

Experimental

\begin{tabular}{lcc}
\hline Ingerido & 4,88 & 4,56 \\
Deposiciones & 0,99 & 0,31 \\
Absorbido & 3,89 & 4,25 \\
\% Absorción & $80 \%$ & $93 \%$ \\
\hline
\end{tabular}

T A B L A 3

BALANCE DE AGUA EN 8 LACTANTES CON DESNUTRICION MARASMICA GRAVE (ml/Kg/día)

\begin{tabular}{lcc}
\hline & Control & Experimental \\
\hline $\begin{array}{l}\text { Alimento } \\
\text { Ingerido: }\end{array}$ & 148 & 114 \\
\multicolumn{1}{c}{ Agua } & 43 & 28 \\
\hline \multicolumn{1}{c}{ T o t a 1 } & 191 & 143 \\
Deposiciones & 11,4 & 13,0 \\
Orina & 77,7 & 37,3 \\
Pérdida Jnsensible & 3,9 & $3,9 \mathrm{ml} / \mathrm{Kg} /$ hora
\end{tabular}

Pérdida insensible calculada según (15) (16).

T A B L A 4

CONCENTRACION URINARIA EN \& LACTANTES CON DESNUTRICION MARASMICA GRAVE

\begin{tabular}{lcccccc}
\hline & \multicolumn{3}{c}{$m E q / 1$} & $g / l$ & \multicolumn{3}{c}{$m E q / 1$} \\
& Dens. & Osm. & N. & Na. & $K$. & $C l$. \\
\hline Control & 1009 & 335 & 4,30 & 21,74 & 36,37 & 31,28 \\
Experim. & 1016 & 621 & 8,83 & 40,78 & 66,11 & 47,48
\end{tabular}


TABLA 5

BALANCE DE NA, KY CL EN 8 LACTANTES CON DESNUTRICION MARASMICA GRAVE

$m E q / \mathrm{Kg} /$ dia Control Exp. Control Exp. Control Exp.

\begin{tabular}{lllllll}
\hline Ingestión & 3,02 & 3,02 & $\mathbf{4 , 5 8}$ & $\mathbf{4 , 3 1}$ & $\mathbf{3 , 9 0}$ & $\mathbf{2 , 8 0}$ \\
Deposiciones & 0,22 & 0,26 & $\mathbf{0 , 7 4}$ & $\mathbf{0 , 8 9}$ & $\mathbf{0 , 2 9}$ & $\mathbf{0 , 1 6}$ \\
Orina & 1,70 & 1,29 & $\mathbf{2 , 8 2}$ & $\mathbf{2 , 2 6}$ & $\mathbf{2 , 2 9}$ & 1,69 \\
Retención & $\mathbf{1 , 0 1}$ & $\mathbf{1 , 4 7}$ & $\mathbf{1 , 0 2}$ & $\mathbf{1 , 1 6}$ & $\mathbf{1 , 3 2}$ & $\mathbf{0 , 9 5}$ \\
\hline
\end{tabular}

\section{Excresión urinaria en creatinina}

Expresada en forma de indice de creatinina (mg. excretados $/ \mathrm{Kg}$. peso $/ 24$ horas) dio promedio de $11,5(10,6-13,1)$ para el período control de $31,1(10,6-12,9)$ para el experirnental.

Si se utiliza la excreción urinaria total en 24 horas para determinar la masa muscular utilizando el coeficiente: $1 \mathrm{~g}$. creatinina excretada representa $20 \mathrm{Kg}$. músculo (14), se obtiene para el peso corporal promedio del período control, 3.655 , una masa muscular promedio de $842,9 \mathrm{~g}$., es decir, un $23 \%$ del peso del cuerpo. En el período experimental con un peso promedio corporal de 4.209 se obtuvo una masa muscular de $938,9 \mathrm{~g}$. 10 que representa un $22 \%$ del peso corporal.

Discustón y comentarios, Del análisis de los resultados se desprende que esta nueva alimentación es perfectamente tolerable para los lactantes. En esta experiencia se utilizaron lactantes desnutridos de tercer grado, seriamente dañados y por lo tanto muy susceptibles a los cambios alimenticios y específicamente muy exigentes en cuanto a los requerimientos nutritivos.

Esta alimentación no sólo demostró ser bien tolerada por ellos, sino que además promovió un crecimiento igual para niños en esta condición, al de la alimentación con leche exclusiva. Efectivamente la ganancia de peso fue mayor que la esperada en niños normales con el mismo peso, fenómeno que siempre ocurre en los desnutridos con alimentaciones cuali y cuantitativamente bien balanceadas. Esta ganancia de peso fue estable y regular. En ningún momento se presentó alteración de las características de las deposiciones, rechazo de la mamadera o anorexia.

En cuanto a los resultados del balance metabólico se destaca nítidamente la mayor retención de Nitrógeno con el uso de la dieta experimental comparada con la del control (significancia de la diferencia: $p<0,009$ ).

Esta significativa mayor retención de nitrógeno sin embargo no se acompaña de una retención igualmente aumentada de Potasio como podría esperarse.
Esta mayor retención se produce con una discreta mayor ingestión de proteínas en el período experimental $(3,8 \mathrm{~g} / \mathrm{Kg} /$ día que en el período control $(3,3 \mathrm{~g} / \mathrm{Kg} /$ día) y una menor eliminación de nitrógeno urinario. La discrepancia de la retención de nitrógeno y potasio crea problemas en la interpretación de la utilización de este Nitrógeno. Porque de aceptar que fue depositado como proteína en tejidos, habría que aceptar que no lo hizo acompañado de Potasio en la proporcion esperada (17) (18) (19) lo que indicaríi un cambio de estructura química del organismo, fenómeno muy difícil de aceptar como valedero con esta dieta.

En este sentido, sin embargo, no crea problema la similitud de ganancia de peso durante la experiencia ya que teóricamente pudo haberse formado tejido proteico en reemplazo de otro tejido (graso, por ejemplo) o agua. Pero aquí se encontró que la medición de la excreción urinaria de creatinina que es un buen índice de la masa muscular (14) reveló una mantención de la proporción de la masa muscular con el peso corporal. La retención de Potasio, como se observa en los resultados, corresponde al crecimiento de la masa muscular equivalente al aumento de peso del $\mathrm{ni}$ ño entre ambos períodos de balance.

Si bien es cierto que ta masa muscular, sólo representa un 22-23\% del peso corporal (lo que es normal para ese peso y edad) (14) cabe pensar que el Nitrógeno pudo haberse depositado en otros órganos. De ser así, sin embargo, habría que aceptar el cambio químico de esos órganos, concepto cuya reserva se hizo notar más arriba, pero que no se puede rechazar con los elementos medidos.

Estas últimas observaciones destacan más nítidamente la discrepancia de retención a que se ha aludido, to que obliga a buscar otras posibilidades. Una de ellas sería que las substancias nitrogenadas pudieran haber sido utilizadas como clementos energéticos, pero de ser así, el Nitrógeno habría sido eliminado en forma de Urea y por lo tanto detectable en la orina con el método de Kjeldhal utilizado (mide el $\mathrm{N}$ total de las muestras). Lo cual no se demostró.

Otra posibilidad scria quc este Nitrógeno hubiera sido retenido temporalmente en los líquidos del organismo en forma de N Ureico, lo que tampoco se demostró, ya que su medición en sangre a la iniciación y término de los balances no demostró variaciones de concentración.

En resumen, en lo que se refiere a metabolismo nitrogenado, se encontró una significativa mayor retención de Nitrógeno, pero el análisis de los parámetros cuantificados no nos permite explicar su utilización.

La diferente distribución del agua con las dos dietas utilizadas se debe exclusivamente a los di- 
ferentes volúmenes ingeridos. Con la dieta control además de recibir mayor cantiđad en la alimentación, ingirieron mayor cantidad de agua como tal, con una pérdida similar por deposiciones, piel y pulmón; es obvio que con la dieta experimental se restringió la cantidad eliminada por el riñón, es así como se eliminó por esta vía sólo un $26 \%$ del agua ingerida contra un $46 \%$ con la dieta control. Este fenómeno determinó una diferencia de concentración notable en la orina, sin que se produjera una diferencia en la eliminación diaria de $\mathrm{Na} \mathrm{K}$ o $\mathrm{Cl}$ expresada en $\mathrm{mEq} / \mathrm{Kg} /$ día.

La concentración osmolar promedio de orina con la dieta experimental puede considerarse que llegó a niveles demasiado altos e indeseables pari estos lactantes.

Al colocar al niño en esta situación su margen de compensación frente a pérdidas extraterrenales es más reducido (diarreas, sudoración). Pero esta situación es fácilmente corregible con sólo administrar agua en forma de agua libre o diluyendo algo más la alimentación.

La diferencia en el porcentaje de absorción de grasas debe ser atribuida a la diferente calidad de aceites agregados a Ja dieta. En el período control se utilizo el aceite de maravilla y raps, en el experimental se utilizó aceite Mazola. (Después de finalizada esta experiencia se agregó aceite Mazola a la dieta control, comprobándose un mcjor coeficiente de absorción con este alimento).

En lo que respecta al balance de $\mathrm{Na}, \mathrm{K}$ y $\mathrm{Cl}$ las diferencias en retención no son significativas, como tampoco lo son las de ingestión. No se observó cambios de concentración de estos elementos en el líquido extracelular en los análisis practicados en la iniciación y terminación del balance. En este aspecto el riñón se demostró como siempre muy flexible a los cambios individuales dc ingestión, reduciendo o aumentando la eliminación segứn las necesidades de mantención de las concentraciones en el liquido extracelular y por lo tanto del intracelular.

El análisis en conjunto de los resultados de csta experiencia nos demuestra que esta nueva alimentación puede substituir perfectamente a la alimentación a base de leche exclusiva. Especialmente cuando se considera que se utilizaron lactantes "i desnutridos de III grado que son Ios más exigentes en cuanto a requerimiento nutritivo.

\section{RESUMEN}

1.- Se ensaya una nueva alimentación para lactantes entre cuyas características destaca que las proténas están suministradas básicamenpor Harina de Soya y que atiliza solamente una pequeña proporción de leche descremada.
2.- Se utitizan ocho lactantes desnutridos de tercer grado, a quienes se alimenta inicialmente. con la diela de recuperación usual de este hospital hasta que demuestren una ganancia de peso regular y consistente por lo menos durante 15 dias. Sólo en este momento se inicia la alimentación con la nueva dieta.

3.- Se practican dos balances metabólicos de 5 dias de duración. El primero al finalizar el período control y el segundo después de por lo menos 15 dias de estar alimentados con la nueva alimentación.

4.- Se comprueba la excelente aceptabilidad y la ganancia de peso regular y constante, con esta nueva alimentación.

5.-- El balance demuestra una retención de $N$ significativamente mayor con la dieta experimental.

6.- Esta mayor retención no se acompaña da una retención paralela de $K$.

Se discuten los posibles significados de este hallazgo.

7.- El balance graso, demuestra una mayor $a b-$ sorción de grasa con la dieta experimental, fenómeno que se atribuye a la diferente calidad de aceite utilizado.

8.- En el balance de agua y minerales se destaca una mayor concentración osmolar de la orina en el periodo experimental. Se atribuye a la menor ingestión de agua que presentaron en este período, lo que determinó una disminución del volumen urinario.

La eliminación total diaria de $\mathrm{Na} \mathrm{K}$ y $\mathrm{Cl}$, sin embargo no sufrió alieraciones, lo que determina que las retenciones de estos elementos sean totalmente normales.

9.- Se concluye que este nuevo alimento puede reemplazar perfectamente a la dieta láctea usual.

\section{SUMMARY}

1.- A new formula for infants is tested, its main feutures arc that the proteins are supplied basically as soy flour and that it contains a very small amount of skimmed milk.

2.- The study is undertaken in eight infants with third degree malnutrition who are fed initially with the usual recovery dist of this hospital until they experiment a consistent increase in weight for at least 15 days. Only in this moment the experimental dist is started. 
3.- Two metabolic balances of 5 days duration are perfomed. The first at the end of the control period and the second, after being at jeast 15 days with the new formula.

4.- An excellent acceptance and a steady and constant weight gaim are verified with the new formula.

5.- Te balance studie shows a significant increase of nitrogen retention with the experimental diet.

6.- Tis increase retention is not accompanied by a paralel retention of $\mathbf{K}$. The significance of this finding is discussed.

7.- The fat balance evidences a greater absortion of fat with the experimental diet, this phenomenon is atributed to differences in quality of the oil used.

8. - In the water and mineral balances, a greater osmolar concentration in the urine during the experimental period is found. This is attribucted to a decreased urinary out put.

However, the total daily output of $\mathrm{Na}, \mathrm{K}$ and $\mathrm{Cl}$ did not change and thus the retention of these elements were completaly normal.

9.- The conclusion is that this new formula can adequately replace the usual milk diet.

\section{Biblografía}

1.-Mönckeberg $F$. Treatment of severe infant malnutrition. Nutrition and Relief Operations in Time of Disaster. Edit. Swedish Nutrition Foundation. Uppsala, 1971.

2.-Möncheberg F., Donosa G., Oxman S. Human Growth in Infantil Malnutrition. Pediatrics 31: 58, 1963.

3.- Märckeberg $F$. Adaptation to chronic caliorie and protein restriction in infants. Protein Calorie Deficiencies and Protein Deficiencias. Ed. McCance and Widdowson. Churchill, London (1967) p. 91.

4.-Gonzalez C., Mönckeber F., Flores H., Reid A. Diversas disacaridasas en la mucosa intestinal del lactatnte desnutrido. Soc. Latinoamericana de Investigación Pediátrica. LX Meeting, Valdivia, Chile p. 41 (1969).

5.-Mönckeberg $F$, Monzur $F$, y López $M$. Algunos factores que influencian la absorción de grasas en el Iactante desnutrido. Pediatria (Chile) 1: 107. 1958.
6.-Flores H., Seakins A, and Mönckeberg F. Mechanism of fatty liver in infantile malnutrition. Intern. Symposium Dietary and post natal Development, 1972. Milán (In Press).

7.-Mönckeberg $F$. Les graisses dans l'alimentation du nourrison. Annales Nestlé 62: 53. 1972.

8.- Ariztía A., Eggers F., Mayersein $G$, and Aspillaga $M$. Tre use of a fat dist in the treatment of malnutrition. Helv. Pead. Acta. 14: 113, 1959.

9.-Jarpa S., Martner G. Estudios en lactantes distroficos. 1. Organización de una Unidad Metabólica. Revista Chilena de Pediatria 33: 7, 1962.

10.-O'Brien, Donough \& Frank A. Ibbott. Laboratory Manual of Pediatric Micro and Ultramicro-Biochemical Techniques Third Edition. Hoeber Medical Division. Harper \& Row, Publishers. Incorporated. New York 1962.

11.-Hare, R. S. Proc. Exp. Biol. and Med. 70: 148, 1950 (citado por 14).

12.- Latson H, D. Appl. Physiol, 4: 227, 1951 (citado por 14).

13.-Metcoff, J. and Antonowicz. Methods of Analysis. Dr. James $L$. Gamble's Laboratory. Department of Pediatrics Harvard. Medical School. Boston 1956.

14. - Cheek, Donal B. Human Growth Body Composition, Call Growth Energy, and Intelligence. Pag. 183-197, Lea \& Febiger. Philadelphia, 1968.

15.- Marther J., Jurpa S., Ariztía A. Estudios en lactantes distróficos IIr Metabolismo del agua y electrolitos. Revista Chilena de Pediatría 34: 121, 1963.

16.- Cheek, "Human Growth". Pag. 449, Lea \& Febiger. Philadelphia 1968.

17.-Gamble J. L. Chemical anatomy, physiology and pathology of extracellular fluid. Pub. Harvard University Press. Cambridge, Massachussetts, 1958.

18.-Dickerson A. W. T. and E. M. Widdowson. Bio. chem. I. 74: 247, 1960 (Citado por 20).

19.-Albanese, A, A, Newer Methods of Nutritional Biochemistry vol. 2, pag. 57. Academic Press, New York, London 1965. 